\title{
Effects of ultraviolet B radiation on (not so) transparent exopolymer particles
}

\author{
E. Ortega-Retuerta ${ }^{1,2, *}$, U. Passow ${ }^{3,4}$, C. M.Duarte ${ }^{5}$, and I. Reche ${ }^{1,2}$ \\ ${ }^{1}$ Departamento de Ecología, Facultad de Ciencias, Universidad de Granada, Granada 18071, Spain \\ ${ }^{2}$ Instituto del Agua, Universidad de Granada, Granada 18071, Spain \\ ${ }^{3}$ Marine Science Institute, University California Santa Barbara, CA 93106, USA \\ ${ }^{4}$ Alfred Wegener Institute for Polar and Marine Research, 27570 Bremerhaven, Germany \\ ${ }^{5}$ Instituto Mediterráneo de Estudios Avanzados (CSIC-UIB), Miquel Marqués 21, 07190 Esporles, Illes Balears, Spain \\ *now at: UPMC Univ Paris 06/CNRS, UMR 7621, LOBB, Observatoire Océanologique, 66651 Banyuls Sur Mer, France
}

Received: 2 July 2009 - Published in Biogeosciences Discuss.: 28 July 2009

Revised: 11 November 2009 - Accepted: 8 December 2009 - Published: 17 December 2009

\begin{abstract}
Transparent exopolymer particles (TEP) are the most ubiquitous gel particles in the ocean and form abiotically from dissolved precursors. Although these particles can accumulate at the ocean surface, being thus exposed to intense sunlight, the role of solar radiation for the assembly and degradation of TEP is unknown. In this study, we experimentally determined the effects of visible and ultraviolet $\mathrm{B}$ (UVB) radiation on (1) TEP degradation (photolysis experiments), (2) TEP assembly from dissolved polymers (photoinhibition experiments) and (3) TEP release by microorganisms. Solar radiation, particularly in the UVB range, caused significant TEP photolysis, with loss rates from 27 to $34 \%$ per day. Dissolved polysaccharides did not increase in parallel. No TEP were formed under UVB, visible or dark conditions, indicating that light does not promote TEP assembly. UVB radiation enhanced TEP release by microorganisms, possibly due to cell deaths, or as a protective measure. Increases in UVB may lead to enhanced TEP photolysis in the ocean, with further consequences for TEP dynamics and, ultimately, sea-air gas exchange.
\end{abstract}

\section{Introduction}

Transparent exopolymer particles (TEP), defined as large, sticky particles, stainable with alcian blue (Alldredge et al., 1993), are the most ubiquitous gel particles in the ocean

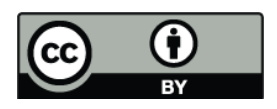

\section{Correspondence to: E. Ortega-Retuerta} (ortega@obs-banyuls.fr)
(Passow, 2002a; Verdugo et al., 2004). TEP are predominantly formed by spontaneous self-assembly of dissolved precursors, mostly acidic polysaccharides that are released by microorganisms (Passow and Alldredge, 1994). TEP facilitate aggregation and carbon export from the surface to deep waters acting as the matrix of large aggregates (Passow et al., 2001; Engel et al., 2004). However, the density of TEP is low and, unballasted, TEP move upward (AzetsuScott and Passow, 2004; Mari, 2008), accumulating in the surface microlayer (SML) (Wurl and Holmes, 2008). This microlayer, about $100 \mu \mathrm{m}$ thick, is located at the air-ocean interface, and is enriched in organic and inorganic matter, especially in surface-active compounds (Sieburth et al., 1976). The SML can severely affect exchange processes between the ocean and atmosphere, including the retardation of airsea gas exchange (Frew, 1997). Relative partitioning of TEP between the aggregated fraction, which sediments and the fraction that accumulates at the sea surface may determine net carbon flux.

To date, the effects of solar radiation on organic matter dynamics have been mostly focused on chromophoric and humic substances. These organic compounds are susceptible to photodegradation to lower molecular weight compounds and inorganic forms (dissolved inorganic carbon, carbon monoxide and dioxide) (Kieber et al., 1990; Mopper et al., 1991; Miller and Zepp, 1995; Reche et al., 1999). In turn, humiclike compounds can be generated by sunlight-mediated condensation of dissolved fatty acids derived from phytoplankton (Kieber et al., 1997; Reche et al., 2001). Despite the potential for phototransformations of TEP, to our knowledge there is no specific information of the effect of solar

Published by Copernicus Publications on behalf of the European Geosciences Union. 
radiation, both in the ultraviolet (UV) and visible ranges, on TEP assembly from their dissolved precursors or vice versa. The polysaccharide content and gel character of TEP confer high transparency to these particles in the visible range (Alldredge et al., 1993; Passow, 2002a) and suggest low photoreactivity in this range (i.e. chemical changes associated with photon absorption).

However, Orellana and Verdugo (2003) found that ultraviolet B (UVB) radiation can block the abiotic self-assembly of dissolved organic polymers into gels, and may completely disperse preexisting gels. The proposed mechanism for this breakage is a loss of anionic charge, which would lead to a decline in the polymer network stability. Moreover, polysaccharides such as alginic acid, pullullan and laminarin can also be photolysed at high UVB levels (Kovac et al., 2000; Akhlaq et al., 1990). We hypothesized that UVB radiation will decrease TEP concentration, both by photolytic dispersion of existing TEP and by inhibiting TEP assembly. High UVB radiation at the surface of the ocean makes this effect especially important for TEP standing stock in the SML, where a reduction in TEP would presumably have significant effects on the gas exchange between the atmosphere and ocean.

In this study, we experimentally tested three hypotheses: first, UVB radiation can disperse pre-assembled TEP into their dissolved precursors (photolysis). Second, UVB radiation can block the self-assembly of dissolved precursors to form TEP (photoinhibition). Third, TEP generation rate by microorganisms is a function of the light exposure.

\section{Material and methods}

\subsection{Experimental set-up}

Different types of experiments were performed to test the three hypotheses (Table 1). North Sea water (collected from two locations at the German Bight, between $53^{\circ}$ and $54^{\circ} \mathrm{N}$ and $7^{\circ}$ and $8^{\circ} \mathrm{E}$ ), was used in four of the experiments, and three were conducted with water from a batch culture of Chaetoceros affinis, a diatom species known to produce large amounts of TEP and their dissolved precursors (Passow and Alldredge, 1994). The culture was grown in $\mathrm{f} / 2$ media based on North Sea water, with a photoperiod of $12 \mathrm{~h}$ light $-12 \mathrm{~h}$ dark at an irradiance of $150 \mu \mathrm{mol}$ photon $\mathrm{m}^{-2} \mathrm{~s}^{-1}$ and harvested in the exponential growth phase. One experiment was conducted with the TEP model substances xanthan gum and alginic acid.

Three experiments were conducted with natural solar radiation by incubating outdoors on the laboratory roof in a water bath (Table 1) with a mean light dose of $0.05 \mathrm{~W} \mathrm{~m}^{-2}$ at UVB, $3.52 \mathrm{~W} \mathrm{~m}^{-2}$ at UVA, and $660 \mu \mathrm{mol}$ photon $\mathrm{m}^{-2} \mathrm{~s}^{-1}$ at PAR. Five experiments were conducted indoors inside a temperature constant culture room $\left(15^{\circ} \mathrm{C}\right)$ under regulated, artificial light regimes with 18:6 h light: dark under UVB
Table 1. Summary of experiments conducted.

\begin{tabular}{llll}
\hline \# & Type of experiment & Sample & Incubation location \\
\hline 0 & TEP photolysis & Model polysaccharides & Laboratory roof \\
1 & TEP photolysis & North Sea water & Culture room \\
2 & TEP photolysis & C. affinis culture & Culture room \\
3 & TEP photolysis & C. affinis culture & Culture room \\
4 & TEP photoinhibition & North Sea water & Laboratory roof \\
5 & TEP photoinhibition & North Sea water & Culture room \\
6 & TEP photoinhibition & C. affinis culture & Culture room \\
7 & TEP photoreactivity & North Sea water & Laboratory roof \\
& with microorganisms & & \\
\hline
\end{tabular}

and PAR lamps, yielding a light dose of $0.15 \mathrm{~W} \mathrm{~m}^{-2}$ at UVB, $5.86 \mathrm{~W} \mathrm{~m}^{-2}$ at UVA, and $10 \mu \mathrm{mol}$ photon $\mathrm{m}^{-2} \mathrm{~s}^{-1}$ at PAR.

\subsubsection{TEP photolysis experiments}

A total of four experiments to determine if TEP photolysis is significant were conducted. Three experiments were conducted inside culture rooms, another smaller experiment (experiment 0 ) that used model polysaccharides was conducted under natural light conditions. Experiment 1 was performed with natural North Sea water and experiments 2 and 3 with the filtrate of $C$. affinis. North Sea water or water from the batch culture of $C$. affinis were sequentially filtered: first through a $40 \mu \mathrm{m}$ mesh, then through precombusted GF/C and GF/F glass fiber filters $(\approx 1.2 \mu \mathrm{m}$ and $\approx 0.7 \mu \mathrm{m}$, Whatman $)$ and then through $0.2 \mu \mathrm{m}$ polycarbonate filters (Poretics). This procedure removed all particles, but filtrates contained (dissolved) TEP-precursors. This filtrate was subsequently incubated in a Couette flocculator for $24 \mathrm{~h}$ at a shear rate of $33.3 \mathrm{~s}^{-1}$ to induce the formation of TEP from dissolved precursors. A Couette flocculator consists of a fixed inner and a rotating outer cylinder which provide a quantifiable 2dimensional laminar shear in the annular space between them (Duuren, 1968). Incubation in a flocculator under these conditions enhances TEP formation from dissolved precursors (Passow, 2000). The resultant TEP-enriched, but organismsfree water was used in the experiments on TEP photolysis. The acidic polysaccharides xanthan gum $\left(3.4 \mathrm{mg} \mathrm{L}^{-1}\right)$ and alginic acid $\left(5.5 \mathrm{mg} \mathrm{L}^{-1}\right)$ were used instead of natural TEP for the exploratory experiment 0 .

Experiments 1 to 3 consisted of three treatments in triplicate: (1) +UVB (water incubated in $50 \mathrm{~mL}$ quartz bottles, transparent to UVB, UVA and PAR light), (2) - UVB (water incubated in $50 \mathrm{~mL}$ borosilicate bottles, opaque to UVB light and transparent to UVA and PAR light), and (3) dark (water incubated in aluminium-covered borosilicate $50 \mathrm{~mL}$ bottles). Experiment 0 consisted of duplicate dark (dark treatments) or light (+UVB treatment) only (no - UVB treatment).

Samples from experiment 1 to 3 were collected initially, after 1.5 and after 3 days to measure concentrations of TEP, dissolved mono- and polysaccharides (DTCHO) 
and bacterial abundance. Bacteria concentration had to be monitored because bacteria generate and utilize TEP (Passow, 2002b; Stoderegger and Herndl, 1999) and may pass throughout $0.2 \mu \mathrm{m}$ filters (Hahn, 2004). Only changes in xanthan gum and alginic acid concentration were monitored in experiment 0 initially and after 3 days.

Normalized TEP photolysis rates were calculated after the following expression:

$-\Delta \operatorname{TEP}\left(\mathrm{d}^{-1}\right)=\frac{\left(\mathrm{TEP}_{t_{\mathrm{f}}}-\mathrm{TEP}_{t_{0}}\right) / \mathrm{t}}{\mathrm{TEP}_{t_{0}}} \cdot 100$

where $t_{\mathrm{f}}$ and $t_{0}$ are final and initial times respectively and $t$ is incubation time in days.

\subsubsection{TEP photoinhibition}

A total of 3 experiments were conducted to assess the role of solar radiation on the self-assembly of dissolved polymers to form TEP. We used natural (experiment 4) or artificial light (experiments 5 and 6), and water from either the North Sea (experiments 4 and 5) or from the $C$. affinis culture (experiment 6). The water was filtered through $0.2 \mu \mathrm{m}$ to eliminate phytoplankton, bacteria and TEP. This $<0.2 \mu \mathrm{m}$ filtrate was used directly to initiate the experiments. Each experiment consisted of the same three treatments (+UVB, $-\mathrm{UVB}$, dark, each in triplicate) as described above. Samples were collected initially, and after 1.5 and 3 days to determine TEP and DTCHO concentration as well as bacterial abundance. We confirmed the presence of dissolved TEP-precursors in the water used for experiments by incubating an aliquot of the $<0.2 \mu \mathrm{m}$ filtered water in a Couette flocculator for 1 day in the dark and determined TEP formation rate.

\subsubsection{Influence of solar radiation on TEP generation in the presence of microorganisms}

Experiment 7 was conducted with unfiltered (whole planktonic community) North Sea water. The experiment consisted of the same three treatments as the prior experiments using the same $250 \mathrm{~mL}$ bottles, with 3 replicates each, and was conducted under natural solar radiation. Samples were collected initially, and after 4.5 and 9 days for the determination of TEP and DTCHO concentrations.

\subsubsection{Methodological tests}

Three types of control experiments were conducted to test our experimental set-up.

First, the light transmission through the borosilicate and quartz bottles, respectively, was measured by placing quartz and borosilicate pieces inside a spectrophotometer. Second, absorbance spectra (250 to $500 \mathrm{~nm})$ of the acidic polysaccharides xanthan gum $\left(3.4 \mathrm{mg} \mathrm{L}^{-1}\right)$ and alginic acid $\left(5.5 \mathrm{~m} \mathrm{~L}^{-1}\right)$ were measured to determine if TEP-like substances absorb in the UV range. The presence of chro- mophoric groups (e.g. double bonds, rings, etc.) is indicative of the susceptibility of an organic compound to react with solar radiation, and absorption in the UV range suggests photoreactivity under UV light. Absorbance was converted to Napierian absorption coefficients in $\mathrm{m}^{-1}$ (Miller, 1998).

Third, loss of TEP due to sticking to bottle walls was a concern, and the potential for TEP to adhere to bottle walls was investigated. We incubated the model substance xanthan gum at a concentration of $1.56 \mathrm{mg} \mathrm{L}^{-1}$, which lies within the range of natural concentrations (Passow, 2002a) on the laboratory roof for three days and measured alcian blue-stainable material attached to bottle walls directly. We prepared three treatments: uncovered quartz bottles (+UVB), quartz bottles covered by aluminium foil (quartz, dark treatment), and borosilicate bottles covered with aluminium foil (borosilicate, dark treatments). Initially and at the end we measured the concentration of gum xanthan (as alcian blue absorbance) in the experimental bottles, and assessed directly the amount of alcian blue stainable material attached to bottle walls. Determination of alcian blue-stainable material on bottle walls was performed by adding two $\mathrm{mL}$ of alcian blue solution to each empty bottle, and swirling the dye carefully repeatedly to ensure contact with all surfaces. Staining of TEP-like substances with alcian blue is immediate. We then rinsed the bottles with MilliQ water to remove excess dye and added five $\mathrm{mL} 80 \%$ sulphuric acid to extract the alcian blue from the organic matter to which it was bound. After $3 \mathrm{~h}$ during which time the bottle was swirled and agitated frequently, we measured the absorbance of the sulphuric acid solution following standard procedures for the colorimetric TEP method (see below).

\subsection{Chemical and biological analyses}

\subsubsection{Transparent exopolymer particles (TEP)}

TEP was determined following the colorimetric method of (Passow and Alldredge, 1995). Briefly, TEP were filtered onto $0.4 \mu \mathrm{m}$ polycarbonate filters, stained with Alcian Blue solution, the filters soaked in $80 \%$ sulphuric acid for $3 \mathrm{~h}$ and measured spectrophotometrically at $787 \mathrm{~nm}$, using empty but stained filters as blanks. Alcian Blue absorption was calibrated using a xanthan gum solution. TEP concentration was expressed in $\mu \mathrm{g}$ of Xanthan Gum equivalents per litre. The detection limit of the method was $2.2 \mu \mathrm{g} \mathrm{XG} \mathrm{eq} \mathrm{L}^{-1}$ and the coefficient of variation was $13 \%$.

\subsubsection{Dissolved Mono (DMCHO) and polysaccharide (DPCHO)}

Samples were filtered through pre-combusted (Whatman $\mathrm{GF} / \mathrm{F}$ ) glassfiber filters and stored in pre-combusted glass scintillation vials at $-20^{\circ} \mathrm{C}$ until analysis. Although $\mathrm{GF} / \mathrm{F}$ filters do not have a well-defined pore-size, the average size after combustion is ca. $0.5 \mu \mathrm{m}$. DMCHO and DPCHO were 
analyzed following the ferricyanide reaction before (DM$\mathrm{CHO}$ ) or after (DPCHO) hydrolysis by oxidation of the free reduced sugars (Myklestad et al., 1997). The reagents and standard solutions were made up in MilliQ water. Reagents were calibrated using a standard curve made of d-glucose, and triplicate reagent blanks in MilliQ water were subtracted. Concentrations were expressed in $\mu \mathrm{mol}$ of glucose carbon equivalents per liter $\left(\mu \mathrm{mol}\right.$ gluc- $\left.\mathrm{CL}^{-1}\right)$. The detection limit of the method was $0.4 \mu \mathrm{mol}$ gluc-C $\mathrm{L}^{-1}$, and the coefficient of variation between samples was $7 \%$.

\subsubsection{Xanthan gum/alginic acid}

Xanthan gum and alginic acid (both SIGMA) were mixed in DI water, homogenized and concentrations measured by filtering known volumes onto preweighed $0.4 \mu \mathrm{m}$ polycarbonate filters that were subsequently dried for $2 \mathrm{~h}$, and reweighed. Xanthan gum and alginic acid concentrations $\left(\mu \mathrm{g} \mathrm{L}^{-1}\right)$ were calculated from the difference of weigh before and after filtration divided by the filtered volume.

\subsubsection{Bacterial abundance}

Bacterial abundance was determined by enumeration using an epifluorescence microscope. Samples of $4-10 \mathrm{~mL}$ were filtered onto a black $0.2 \mu \mathrm{m}$ polycarbonate Filter (Poretics) and stained with DAPI (4,6-diamidino-2 phenylindole) to a final concentration of $1 \mu \mathrm{g} \mathrm{mL}-1$. Slides were stored frozen. At least 350 cells in 15 random fields were counted (Porter and Feig, 1980).

\subsection{Statistical analyses}

To test for significant differences between treatments over time in each experiment, repeated measures ANOVA tests were applied. This analysis is useful for experiments where there may be a within-treatment effect (incubation time) and a between-treatment effect (+UVB, -UVB or dark) (Sokal and Rohlf, 1995). The analysis tests three types of effects allowing separating three hypotheses: within-treatment effect (does TEP or DTCHO change through incubation time?), between-treatment effect (are TEP or DTCHO different in +UVB, $-\mathrm{UVB}$ or dark treatments at every incubation time?), and between-treatment by within-treatment interactions (does the difference between TEP in +UVB, $-\mathrm{UVB}$ and dark treatments increase through incubation time?).

\section{Results}

\subsection{TEP photolysis experiments}

TEP concentration decreased markedly in the +UVB treatments of all the photolysis experiments. TEP concentrations had decreased after 1.5 days and continued to decrease till the end of each experiment (3 days), resulting in undetectable
Table 2. Results of repeated measures ANOVA performed to assess significant differences in TEP concentrations among the different treatments of TEP photolysis experiments (exp. 1 to 3 ). As experiment 0 was a small test experiment only (not all treatments, duplicates rather than triplicates) it was not included in the statistical analysis. Ns=not significant. Bold: significant.

\begin{tabular}{llrr}
\hline Experiment & Effect & F & $p$ level \\
\hline 1 & Full solar spectrum (UVB vs. Dark) & 6.3 & $\mathrm{~ns}$ \\
& Full sol. sp.xTime & 2.15 & $\mathrm{~ns}$ \\
& UVB (+UVB vs. -UVB) & 32.11 & $<0.05$ \\
& UVBxTime & 1.74 & $\mathrm{~ns}$ \\
& -UVB (-UVB vs. Dark) & 5.44 & $\mathrm{~ns}$ \\
& -UVB - Time & 1.74 & $\mathrm{~ns}$ \\
& Full solar spectrum (UVB vs. Dark) & 23.6 & $<0.01$ \\
& Full sol. sp. - Time & 7.3 & $<0.05$ \\
& UVB (+UVB vs. -UVB) & 3.7 & $\mathrm{~ns}$ \\
& UVB - Time & 1.2 & $\mathrm{~ns}$ \\
& -UVB (-UVB vs. Dark) & 7.3 & $\mathrm{~ns}$ \\
& -UVB - Time & 2.9 & $\mathrm{~ns}$ \\
& Full solar spectrum (UVB vs. Dark) & 509.0 & $<0.01$ \\
& Full sol. sp. - Time & 108.9 & $<0.01$ \\
UVB (+UVB vs. - UVB) & 367.4 & $<0.01$ \\
& UVBxTime & 11.9 & $<0.05$ \\
& -UVB (-UVB vs. Dark) & 47.8 & $\mathrm{~ns}$ \\
& -UVBxTime & 23.2 & $<0.05$ \\
\hline
\end{tabular}

concentrations in 2 out of 3 experiments. When UVB was excluded (-UVB) TEP decreased in experiment 2 only, and at lower rates. No changes (exp. 1) or increases in TEP (exp. 2 and 3) were detected in the dark treatments (Fig. 1). Repeated measures ANOVA confirmed differences between $+\mathrm{UVB}$ and -UVB or dark treatments to be statistically significant (Table 2). Results of experiment 0 confirm the distinct decrease in the concentrations of acidic polysaccharides during the 3 day incubation in the light (+UVB) (Fig. 2). In dark treatments, concentration of both model polysaccharides did not change noticeably during the incubation, suggesting loss processes to be unimportant in the dark.

Bacterial growth was low in +UVB and -UVB treatments, ranging from undetectable to $0.02 \times 10^{9}$ cells $\mathrm{L}^{-1}$ in $+\mathrm{UVB}$ treatments and to $0.3 \times 10^{9}$ cells L $^{-1}$ in $-\mathrm{UVB}$ treatments. However, high bacterial growth was detected in the dark treatments, particularly in those experiments performed with culture filtrate (exps. 2 and 3) with bacterial concentrations reaching up to $11.4 \times 10^{9}$ cells $\mathrm{L}^{-1}$ in three days (exp. 3 dark treatment). As bacteria are able to generate TEP (Passow, 2002 b), we estimated the potential contribution of bacteria to TEP production using an equation empirically obtained from incubation experiments on the production of TEP by bacteria (Ortega-Retuerta, 2008):

$$
\begin{aligned}
& \Delta \mathrm{TEP}\left(\mu \mathrm{gXGeqL} \mathrm{L}^{-1} \mathrm{~d}^{-1}\right) \\
& \quad=5.73+5.37 \times 10^{-8} \Delta \mathrm{BA}\left(\text { cell L }^{-1} \mathrm{~d}^{-1}\right)
\end{aligned}
$$


Table 3. TEP photolysis rates $\left(\mathrm{d}^{-1}\right)$ determined in $+\mathrm{UVB}$ and -UVB treatments after corrections by bacterial growth.

\begin{tabular}{ccc}
\hline \multirow{2}{*}{ Experiment } & \multicolumn{2}{c}{ Photolysis rate $\left(\%, \mathrm{~d}^{-1}\right)$} \\
& UVB & -UVB \\
\hline 0 & $22-27$ & No data \\
1 & $27 \pm 5$ & $10 \pm 4$ \\
2 & 34 & $18 \pm 2$ \\
3 & 32 & $8 \pm 8$ \\
\hline
\end{tabular}
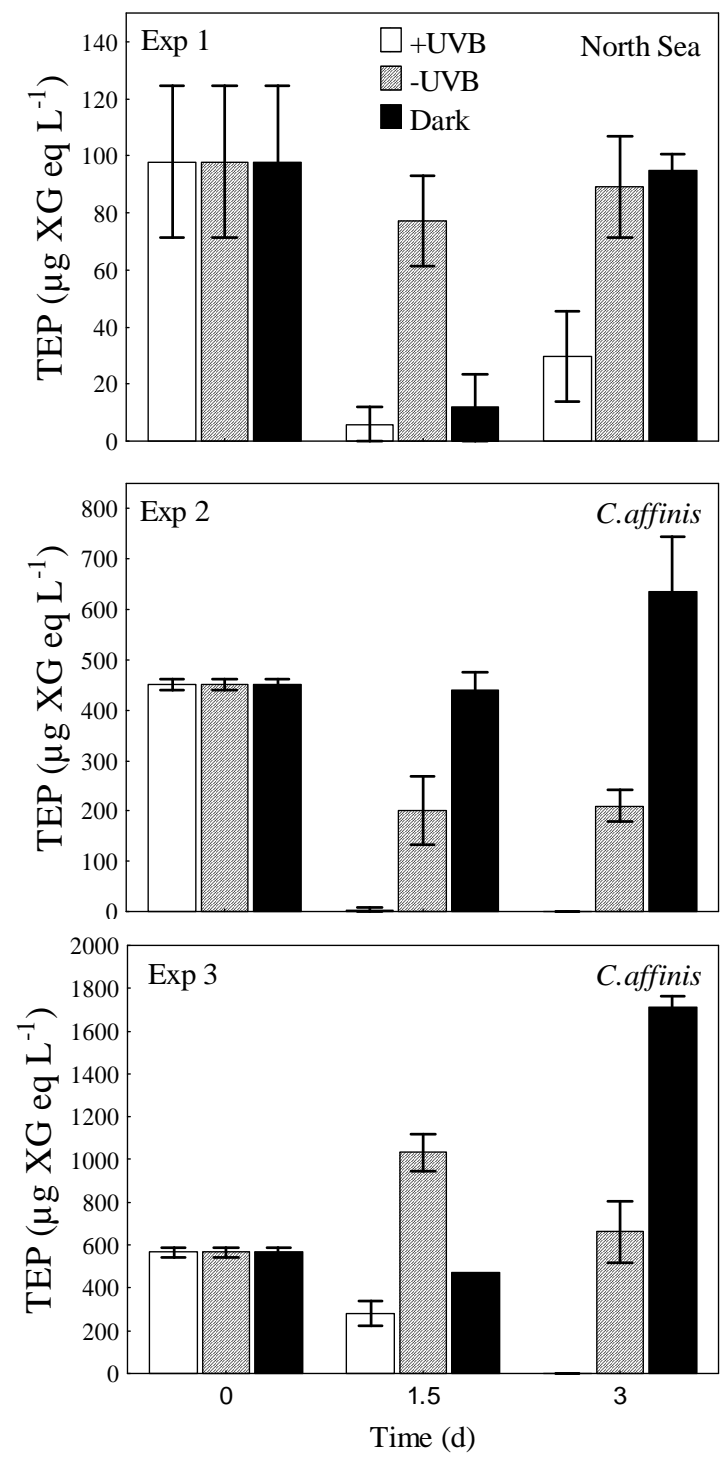

Fig. 1. Changes in TEP concentrations over incubation in the +UVB (white bars), -UVB (grey bars) and dark (black bars) treatments of photolysis experiments 1 to 3 before correcting for bacterial contributions. Whisker $=$ Standard Error of triplicates.

Correction for the potential bacterial production of TEP did not change the main results: the net decrease in TEP was appreciable in +UVB treatments of photolysis experiments

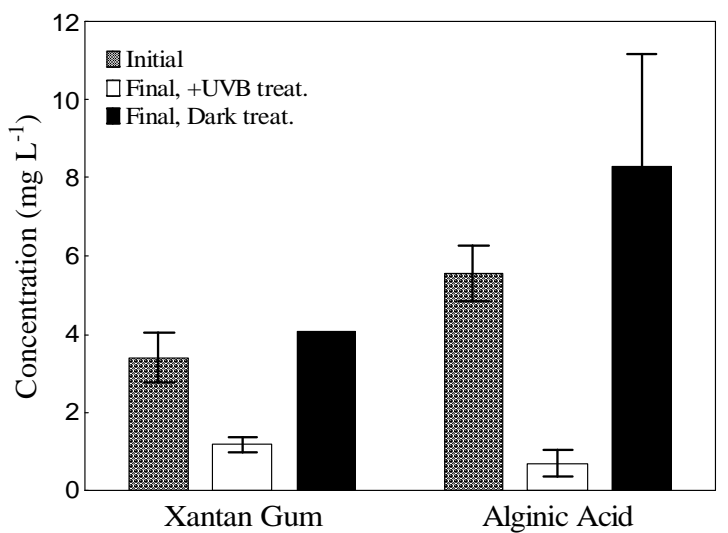

Fig. 2. Photolysis experiment 0: concentration of xanthan gum (left columns) and alginic acid (right columns) at time 0 (hatched bars), in +UVB after 3 days (white bars) and in dark controls after 3 days (black bars). Whiskers $=$ Standard Error of duplicates.

(1-3) and in -UVB treatments of experiments 1 and 2, with no changes in TEP in experiment 3 . In the dark treatments, after the correction for bacterial contributions, no significant changes were detected in TEP concentration, suggesting that the increase in TEP in dark treatments was due to bacterial activity.

Bacteria are also able to utilize TEP, but no correction was deemed necessary for loss due to bacteria, as bacteria concentrations increased in dark treatments, where TEP concentrations increased, rather than decreased.

In our experiments, time averaged TEP photolysis rates ranged from 27 to $34 \% \mathrm{~d}^{-1}$ in the +UVB treatments. However, photolysis rates in experiments 1 and 2 were 69 to $71 \%$ during the first 1.5 days, leading to almost undetectable TEP concentrations, whereas this rate was constant (32\%) during the whole 3 days in experiment 3 (Table 3 ). Photolysis rate of xanthan gum and alginic acid were $22 \%$ and $27 \% \mathrm{~d}^{-1}$. In the -UVB treatments TEP photolysis rates ranged from negligible $\left(8 \pm 8 \% \mathrm{~d}^{-1}\right)$ to $18 \pm 2 \% \mathrm{~d}^{-1}$.

Dissolved mono- and polysaccharide (DTCHO) concentrations also decreased in the +UVB treatments of all experiments, but were statistically significant only for +UVB treatment of experiments $2(p<0.001)$ and $3(p<0.05)$ (Fig. 3$)$. In contrast, there was no consistent pattern in the -UVB and dark treatments. DTCHO photolysis rates in the +UVB treatments ranged from $2.3 \pm 0.5$ to $17 \pm 0.2 \% \mathrm{~d}^{-1}$.

\subsection{TEP photoinhibition experiments}

TEP formation was low or not detectable in all treatments of experiments 4, 5 and 6 leading to non-significant differences in concentrations between treatments or over time ( $p>0.05$, Fig. 4). The flocculator test confirmed the presence of dissolved TEP precursors in the samples, as a significant amount of TEP (between 100 and $1100 \mu \mathrm{g} \mathrm{XG} \mathrm{eq} \mathrm{L}{ }^{-1}, p<0.001$ ) was 

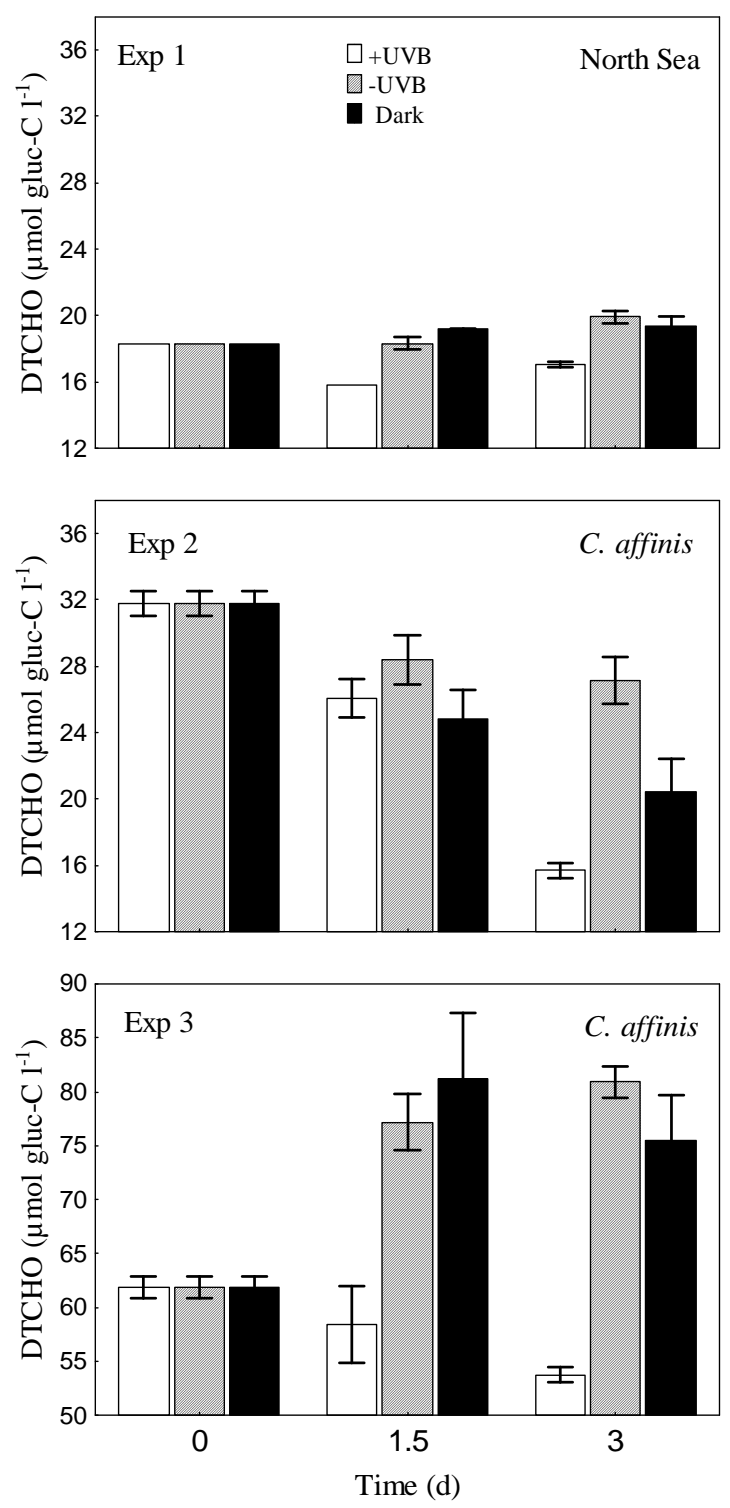

Fig. 3. Changes in DTCHO concentrations over incubation in the +UVB (white bars), -UVB (grey bars) and dark (black bars) treatments of photolysis experiments 1 to 3 . Whisker $=$ Standard Error of triplicates.

formed within $24 \mathrm{~h}$ in the water used for experiments 4 and 5 . This test was not performed in experiment 6 due to the low volume of water available.

DTCHO generally decreased in all photoinhibition experiments and treatments, except for the +UVB treatment of experiment 4 . The differences between treatments, like for TEP concentration, were not statistically significant $(p>0.05)$. Bacteria did not show measurable growth in any treatment of experiments 4,5 or 6 , except for the dark treatment of experiment 6 , where bacteria reached an abundance of $32 \times 10^{6}$ cells $\mathrm{L}^{-1}$.
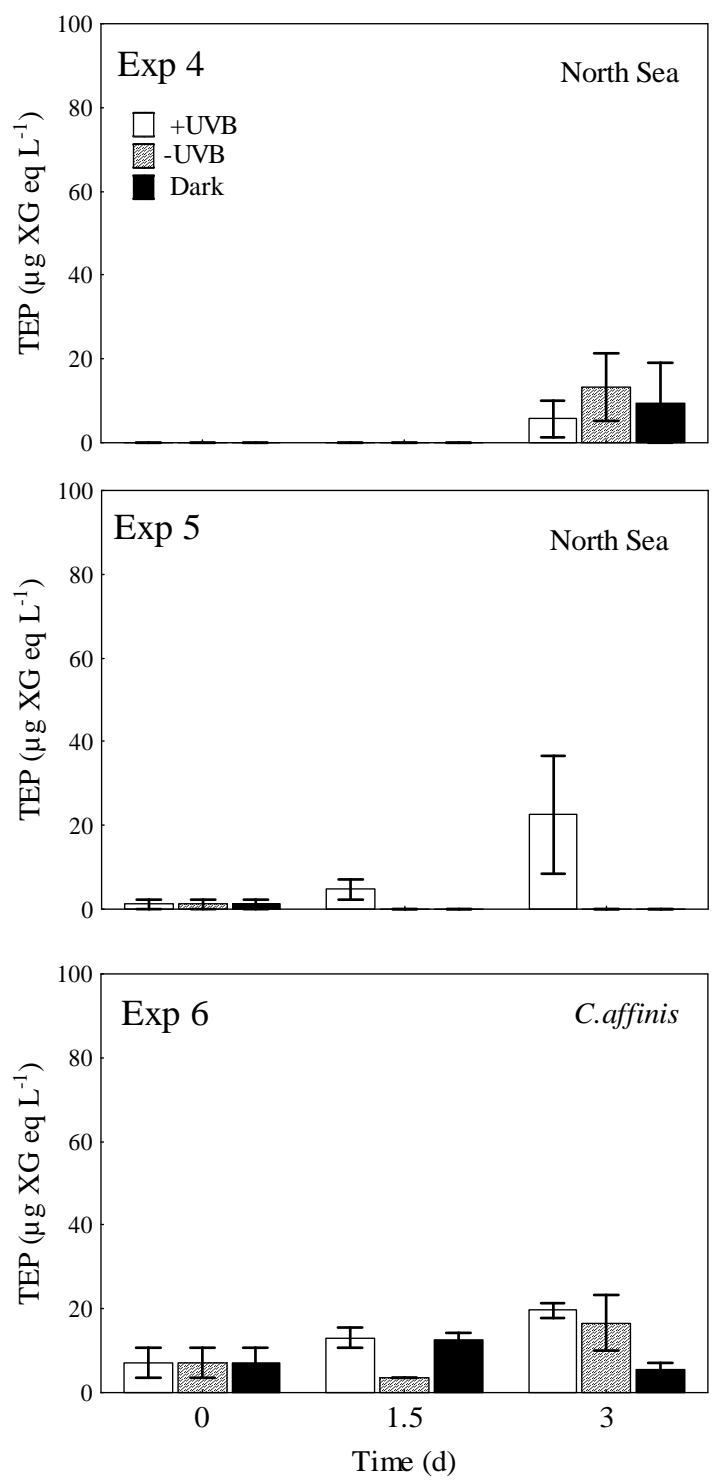

Fig. 4. Changes in TEP concentrations over incubation in the $+U V B$ (white bars), -UVB (grey bars) and dark (black bars) treatments of photoinhibition experiments 4 to 6 . Whisker $=$ Standard Error of triplicates.

\subsection{Influence of solar radiation on TEP generation in the presence of microorganisms}

In experiment 7, high increases in TEP and DTCHO (Fig. 5) were observed over the incubation period, particularly in $+\mathrm{UVB}$ and -UVB treatments. TEP increased a of $17 \%$ and $11 \% \mathrm{~d}^{-1}$ in $+\mathrm{UVB}$ and - UVB treatments, respectively. Increases in TEP were significantly higher in the +UVB treatments, while increases in DTCHO were higher in the -UVB treatments (Table 4). 

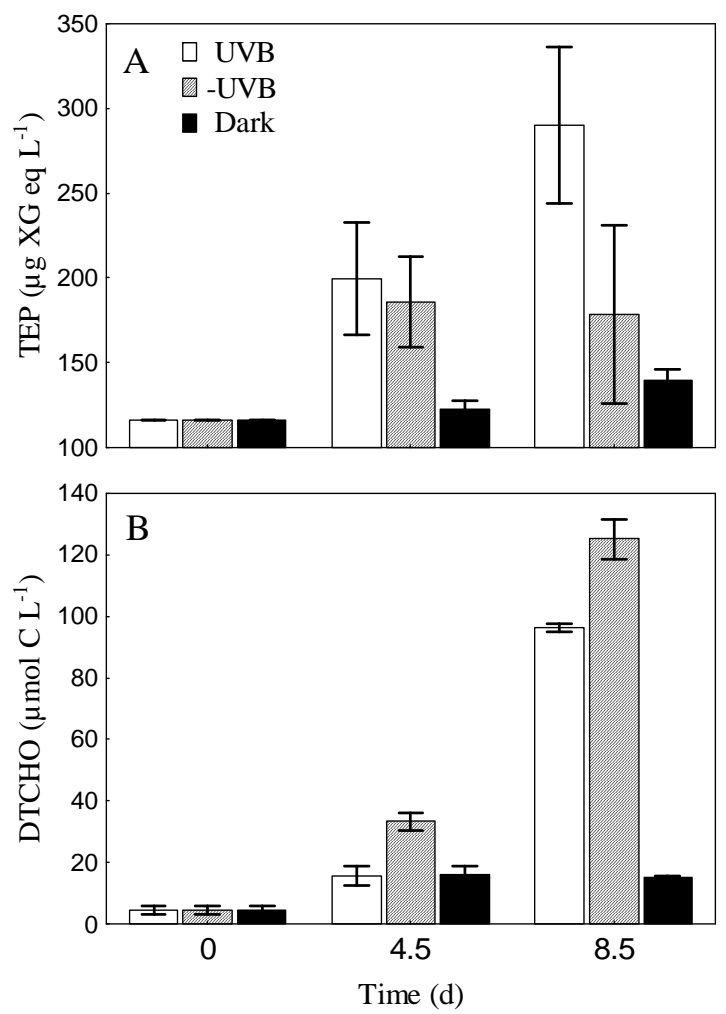

Fig. 5. Changes in TEP (A) and DTCHO (B) concentrations: +UVB (white columns), -UVB (grey columns) and dark (black columns) treatments of the TEP formation experiment with microorganisms (exp. 7). Whisker= Standard Error of triplicates.

\subsection{Results of methodological tests}

The transmission through the quartz bottles was around $90 \%$ in the whole spectra, whereas through the borosilicate bottles it was zero in the UVB range and over $60 \%$ in the UVA and PAR ranges (Fig. 6a). Thus borosilicate bottles effectively removed all UVB radiaton, but also reduced the amount of PAR and UVA light slightly compared to quarz bottles. However, only the presence or absence of effects, not the quantitative relationship to the amount of light was investigated.

Xanthan gum and alginic acid, which were used in methodological tests, have been used as model substances for TEP in the past (Passow and Alldredge, 1995). The second experiment showed that although they are transparent in the visible range, both compounds exhibited significant absorption in the UV range (Fig. 6b), implying by extension that UV light can potentially effect the concentration of TEP in the ocean.

To investigate if attachment of TEP to container walls was a loss process of significance in our photolysis experiment, we conducted a methodological experiment measuring alcian blue stainable material on bottle walls directly. We found that the amount of alcian blue stainable material lost to bottle walls constituted $6 \% \pm 2 \%, 7 \% \pm 0 \%$ and $11 \% \pm 3 \%$ of ini-
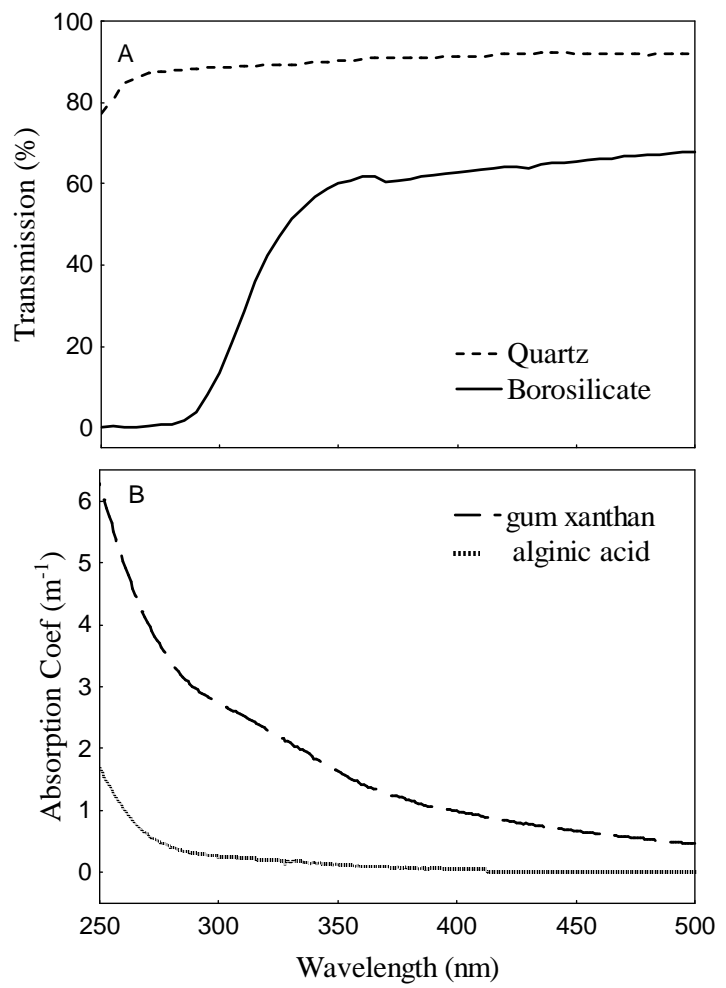

Fig. 6. Results of methodological tests: (A) percentage of light transmission through the experimental bottles for +UVB and -UVB treatments (quartz and borosilicate) as a function of wavelength measured on a spectrophotometer. (B) Absorption spectra from 250 to $500 \mathrm{~nm}$ of xanthan gum and alginic acid solutions.

Table 4. Results of the repeated measures ANOVA for differences in TEP and DTCHO among treatments in experiment 7 (TEP photoreactivity with microorganisms). Ns=not significant. Bold=significant.

\begin{tabular}{|c|c|c|c|}
\hline Variable & Effect & $\mathrm{F}$ & $\mathrm{p}$ level \\
\hline \multirow[t]{6}{*}{ TEP } & Full solar spectrum (UVB vs. Dark) & 42.9 & $<0.01$ \\
\hline & Full sol. sp. · Time & 16.5 & $<0.01$ \\
\hline & UVB (+UVB vs. - UVB) & 6.5 & ns \\
\hline & UVB · Time & 0.8 & ns \\
\hline & -UVB (-UVB vs. Dark) & 17.3 & $<0.05$ \\
\hline & -UVB $\cdot$ Time & 1.9 & ns \\
\hline \multirow[t]{6}{*}{ DTCHO } & Full solar spectrum (UVB vs. Dark) & 71910 & $<0.001$ \\
\hline & Full sol. sp. · Time & 160.2 & $<0.001$ \\
\hline & UVB (+UVB vs. - UVB) & 82.7 & $<0.05$ \\
\hline & UVB $\cdot$ Time & 7.2 & $<0.05$ \\
\hline & -UVB (-UVB vs. Dark) & 613.0 & $<0.01$ \\
\hline & -UVB $\cdot$ Time & 124.4 & $<0.01$ \\
\hline
\end{tabular}

tial TEP concentrations in the +UVB, the dark quarz and the dark borosilicate bottles, respectively. In the +UVB treatment about $6 \%$ of the total loss of TEP during the three day experiment could be attributed to loss to cell walls, whereas this value was higher in the dark treatments $(13 \%$ in quarz bottles and $24 \%$ in borosilicate bottles). 


\section{Discussion}

In this study, we report the first evidence of photolysis of TEP due to UVB light. The particular role of UVB radiation on TEP fragmentation or loss of integrity is consistent with the only published work on the effects of UV radiation on micro-gels (Orellana and Verdugo, 2003). Inhibition of gel assembly likely results from the destabilization of polymer networks caused by a loss of net charge. In that study, full dispersion of self-assembled gels was detected in less than 12 hours when exposed to UVB radiation at $0.5 \mathrm{~W} \mathrm{~m}^{-2}$ that is, a light dose $70 \%$ higher than those in our study $\left(0.15 \mathrm{~W} \mathrm{~m}^{-2}\right)$. We obtained an average TEP photolysis rate of $31 \% \mathrm{~d}^{-1}$, which would yield complete TEP photolysis in ca. 3 days under UVB radiation. However, TEP photolysis rates were even faster, at least $69-71 \%$ per day in 2 of our experiments, leading to a complete loss of TEP in around $35 \mathrm{~h}$ or less. Although UVB radiation appears to dominate photolysis, UVA and PAR radiation also caused TEP photolysis to some extent, with rates up to $17 \%$ per day (Table 3 ). We argue that photolysis was the main loss process of TEP in our experiments (Fig. 2) as bacteria which can either degrade or generate TEP did not grow noticeably in the +UVB and - UVB treatments. Moreover, our tests showed that loss of TEP due to adhesion to bottle walls was small compared to overall loss (about 7\% in +UVB). Consequently, our results indicate that photolysis can at times be a significant loss process of TEP, which should be included in future TEP budgets and carbon cycling scenarios. Other known loss processes of TEP include sedimentation (Passow et al., 2001) or uptake of colloidal organic matter by flagellate grazing (Passow and Alldredge, 1994; Tranvik et al., 1993).

DTCHO generally decreased in the +UVB treatments of photolysis experiments, indicating that DTCHO were not the final products of TEP photolysis. TEP photolysis products could be other dissolved organic compounds or, alternatively, dissolved inorganic carbon forms. Photoproduction of inorganic carbon after irradiation of organic matter has been previously reported (Miller and Zepp, 1995; Mopper et al., 1991), and it would imply, therefore, a net loss of organic carbon for the system. Polysaccharides can also be photodegraded into monosaccharides or photomineralized into inorganic forms after the cleavage of glycosidic linkages (Kovac et al., 2000). However, the photolysis rates of DTCHO in our experiments (up to 17\%) were smaller than those of TEP (27-34\%, Table 3), suggesting that the surface active nature of TEP make these particles more susceptible to photolysis compared to the overall pool of polysaccharides.

No significant increases in TEP were observed in any of the treatments of the photoinhibition experiments. As we detected no TEP assembly in dark treatments, we were unable to determine if UVB inhibits the self-assembly of dissolved polymers to TEP. We can state, however, that solar radiation does not promote the abiotic self-assembly of TEP from its precursors, because the lack of TEP assembly in all treatments was not attributable to the absence of a sufficient amount of precursors in the filtered water. TEP were formed abiotically within the same water when submitted to shear. Previous studies (Mopper et al., 1995; Zhou et al., 1998; Passow, 2000) have demonstrated that freshly released precursors abiotically form larger colloids, and ultimately TEP, within hours to days, that is, in shorter incubation times than used in our experiments, in some cases even in the absence of bubbling or shear. However, it seems that in our samples TEP did not form in the absence of shear independent of light regime.

In the experiment testing TEP formation in the presence of microorganisms, TEP increased greatly under UVB radiation suggesting that UV promotes the production of TEP by organisms. The increase in TEP concentration was higher in +UVB than in -UVB treatments. The higher TEP production under UVB observed in our study could be a consequence of cell disintegration, as UVB often inhibits photosynthesis (Cullen et al., 1992; Lesser, 1996) and causes cell mortality and lysis (Agustí and Llabrés, 2007). Alternatively, the increased release of TEP due to UVB could be a protective measure; TEP may reduce penetration depths of UVB acting similar to the UV-absorbing mycosporinelike amino acids (MAAs) synthesized from macroalgae (e.g. Hoyer, 2003). The gelatinous sea surface microlayer is enriched in bacteria and other organisms (Joux et al, 2006; Cuncliffe and Morrell, 2009) so their release of TEP in this layer is likely of great importance. The influence of UVB light on the release of TEP by organisms, suggested for the first time in this study, deserves further attention.

\section{Conclusions}

We found TEP photolysis due to UVB radiation to be a significant loss process. Non-ballasted TEP migrate upward (Azetsu-Scott and Passow, 2004; Mari, 2008), accumulating in the surface microlayer (Wurl and Holmes, 2008), where they will be exposed to intense solar radiation. Photolysis of TEP at the sea surface may gain further importance in the future ocean, as increasing atmospheric $\mathrm{CO}_{2}$ concentrations lead to increased TEP formation but also result in an increase in the fraction of TEP accumulating at the sea surface (Mari, 2008), where, as our new results imply, TEP will be lost rapidly due to photolysis. This scenario conflicts with the postulated future increase in sedimentation rate due to increased TEP production (Riebesell et al., 2007) predicting a much grimmer picture of the future. Our results combined with those of Mari (2008) suggest that sedimentation rates, and thus the efficiency of the biological pump, could decrease, despite additional TEP formation due to elevated $\mathrm{CO}_{2}$. Ozone depletion (Madronich, 1992) may further magnify this process. Increased loss rates of TEP in the surface layer due to increased UVB radiation could lead to a more 
rapid decrease in TEP concentration at the sea surface, thus impacting gas exchange between the ocean and atmosphere.

Acknowledgements. We gratefully acknowledge the captain and crew of R/V Utörn for their assistance in collecting North Sea water, E. Acero for providing us $C$. affinis cultures and S. Steigenberger and J. Llinas del Torrent for their help with experimental set-up. We also thank the associate editor and two anonymous reviewers for comments in a previous version of this MS. This work was funded by Alfred Wegener Institute for Polar and Marine Research and by the Spanish Ministry of Science and Technology (DISPAR, CGL2005-00076 to IR). E. O.-R. was supported by fellowships of the the Spanish Ministry of Science and Education and University of Granada.

Edited by: J. Toporski

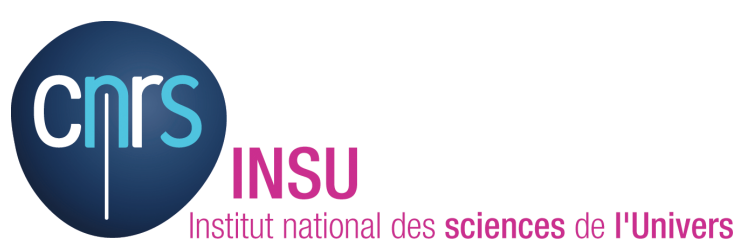

The publication of this article is financed by CNRS-INSU.

\section{References}

Agustí, S. and Llabrés, M.: Solar radiation-induced mortality of marine pico-phytoplankton in the oligotrophic ocean, Photochem. Photobiol., 83, 793-801, 2007.

Akhlaq, M. S., Schuchmann, H. P., and Vonsonntag, C.: Degradation of the polysaccharide alginic acid - a comparison of the effects of UV light and ozone, Environ. Sci. Technol., 24, 379383, 1990.

Alldredge, A. L., Passow, U., and Logan, B. E.: The abundance and significance of a class of large, transparent organic particles in the ocean, Deep-Sea Res. I, 40, 1131-1140, 1993.

Azetsu-Scott, K. and Passow, U.: Ascending marine particles: Significance of transparent exopolymer particles (TEP) in the upper ocean, Limnol. Oceanogr., 49, 741-748, 2004.

Cullen, J. J., Neale, P. J., and Lesser, M. P.: Biological weighting function for the inhibition of phytoplankton photosynthesis by ultraviolet radiation, Science, 258, 646-650, 1992.

Cunliffe, M. and Murrell, J. C. The sea-surface microlayer is a gelatinous biofilm, ISME Journal, 3, 1001-1003, 2009.

Duuren, F. A.: Defined velocity gradient model flocculator, Journal of Sanitary Ingeneering Division ASCE., 94, 671-682, 1968.

Engel, A., Thoms, S., Riebesell, U., Rochelle-Newall, E., and Zondervan, I.: Polysaccharide aggregation as a potential sink of marine dissolved organic carbon, Nature, 428, 929-932, 2004.

Frew, N. M.: The role of organic films in air-sea gas exchange in: The sea surface and global change, edited by: Liss, P. S. and Duce, R. A., Cambridge University Press, Cambridge, 121-173, 1997.

Hahn, M. W.: Broad diversity of viable bacteria in "sterile" $(0.2 \mu \mathrm{m})$ filtered water, Res. Microbiol., 155, 688-691, 2004.
Hoyer, K.: Occurrence, induction and physiological importance of UV-absorbing substances in polar macroalgae, Berichte zur Polar- und Meeresforschung, 440, 1-25, 2003.

Joux, F., Agogue, H., Obernosterer, I., Dupuy, C., Reinthaler, T., Herndl, G. J., and Lebaron, P.: Microbial community structure in the sea surface microlayer at two contrasting coastal sites in the northwestern Mediterranean Sea, Aquat. Microb. Ecol., 42, 91-104, 2006.

Kieber, R. J., Zhou, X. L., and Mopper, K.: Formation of carbonyl compounds from UV-Induced photodegradation of humic substances in natural waters: Fate of riverine carbon in the sea, Limnol. Oceanogr, 35, 1503-1515, 1990.

Kieber, R. J., Hydro, L. H., and Seaton, P. J.: Photooxidation of triglycerides and fatty acids in seawater: Implication toward the formation of marine humic substances, Limnol. Oceanogr, 42, 1454-1462, 1997.

Kovac, N., Bajt, O., and Sket, B.: Photocatalyzed degradation of water soluble polysaccharides, Fresenius Environ. Bull., 9, 217224, 2000.

Lesser, M. P.: Elevated temperatures and ultraviolet radiation cause oxidative stress and inhibit photosynthesis in symbiotic dinoflagellates, Limnol. Oceanogr., 41, 271-283, 1996.

Madronich, S.: Implications of recent total atmospheric ozone measurements for biologically active ultraviolet radiation reaching the Earth's surface, Geophys. Res. Lett., 19, 37-40, 1992.

Mari, X.: Does ocean acidification induce an upward flux of marine aggregates?, Biogeosciences, 5, 1023-1031, 2008, http://www.biogeosciences.net/5/1023/2008/.

Miller, W. L. and Zepp, R. G.: Photochemical production of dissolved inorganic carbon from terrestrial organic matter: Significance to the oceanic organic carbon cycle, Geophys. Res. Lett., 22, 417-420, 1995.

Miller, W. L.: Photochemical principles and experimental considerations, in: Aquatic Humic Substances: Ecology and Biogeochemistry, edited by: Hessen, D. O. and Tranvik, L. J., Springer, Berlin Heidelberg New York, 125-143, 1998.

Mopper, K., Zhou, X. L., Kieber, R. J., Kieber, D. J., Sikorski, R. J., and Jones, R. D.: Photochemical degradation of dissolved organic carbon and its impact on the oceanic carbon cycle, Nature, 353, 60-62, 1991.

Mopper, K., Zhou, J., Ramana, K. S., Passow, U., Dam, H. G., and Drapeau, D. T.: The role of surface-active carbohydrates in the flocculation of a diatom bloom in a mesocosm, Deep-Sea Res. II, 42, 47-73, 1995.

Myklestad, S., Skanoy, E., and Hestmann, S.: A sensitive and rapid method for analysis of dissolved mono-and polysaccharides in seawater, Mar. Chem., 56, 279-286, 1997.

Orellana, M. V. and Verdugo, P.: Ultraviolet radiation blocks the organic carbon exchange between the dissolved phase and the gel phase in the ocean, Limnol. Oceanogr., 48, 1618-1623, 2003.

Ortega-Retuerta, E.: Organic matter distribution and dynamics in marine ecosystems: Field, experimental and remote sensing approaches, Ecology, PhD thesis, University of Granada, Granada, 289 pp., 2008.

Passow, U. and Alldredge, A. L.: Distribution, size and bacterial colonization of transparent exopolymer particles (TEP) in the ocean, Mar. Ecol. Prog. Ser., 113, 185-198, 1994.

Passow, U. and Alldredge, A. L.: A dye-binding assay for the spectrophotometric measurement of transparent exopolymer particles 
(TEP), Limnol. Oceanogr., 40, 1326-1335, 1995.

Passow, U.: Formation of transparent exopolymer particles, TEP, from dissolved precursor material, Mar. Ecol. Prog. Ser., 192, 1-11, 2000.

Passow, U., Shipe, R. F., Murray, A., Pak, D. K., Brzezinski, M. A., and Alldredge, A. L.: The origin of transparent exopolymer particles (TEP) and their role in the sedimentation of particulate matter, Cont. Shelf Res., 21, 327-346, 2001.

Passow, U.: Transparent exopolymer particles (TEP) in aquatic environments, Prog. Oceanogr., 55, 287-333, 2002a.

Passow, U.: Production of transparent exopolymer particles (TEP) by phyto- and bacterioplankton, Mar. Ecol. Prog. Ser., 236, 1-12, 2002b.

Porter, K. G. and Feig, Y. S.: The use of DAPI for identifying and counting aquatic microflora, Limnol. Oceanogr., 25, 943-948, 1980.

Reche, I., Pace, M. L., and Cole, J. J.: Relationship of trophic and chemical conditions to photobleaching of dissolved organic matter in lake ecosystems, Biogeochem., 44, 259-280, 1999.

Reche, I., Pulido-Villena, E., Conde-Porcuna, J. M., and Carrillo, P.: Photoreactivity of dissolved organic matter from high-mountain lakes of Sierra Nevada, Spain, Arct. Antarct. Alp. Res., 33, 426434, 2001.

Riebesell, U., Schulz, K. G., Bellerby, R. G. J., Botros, M., Fritsche, P., Meyerhofer, M., Neill, C., Nondal, G., Oschlies, A., Wohlers, J., and Zollner, E.: Enhanced biological carbon consumption in a high $\mathrm{CO}_{2}$ ocean, Nature, 450, 545-548, 2007.
Sieburth, J. M. N., Willis, P. J., Johnson, K. M., Burney, C. M., Lavoie, D. M., Hinga, K. R., Caron, D. A., French, F. W., Johnson, P. W., and Davis, P. G.: Dissolved organic matter and heterotrophic microneuston in surface microlayers of North Atlantic, Science, 194, 1415-1418, 1976.

Sokal, R. R. and Rohlf, F. J.: Biometry: the principles and practice of statistics in biological research, 3rd edition, edited by: Co., W. H. F. A., New York, 887 pp., 1995.

Stoderegger, K. E. and Herndl, G. J.: Production of exopolymer particles by marine bacterioplankton under contrasting turbulence conditions, Mar. Ecol. Prog. Ser., 189, 9-16, 1999.

Tranvik, L. J., Sherr, E. B., and Sherr, B. F.: Uptake and utilization of colloidal DOM by heterotrophic flagellates in seawater, Mar. Ecol. Prog. Ser., 92, 301-309, 1993.

Verdugo, P., Alldredge, A. L., Azam, F., Kirchman, D. L., Passow, U., and Santschi, P. H.: The oceanic gel phase: a bridge in the DOM-POM continuum, Mar. Chem., 92, 67-85, 2004.

Wurl, O. and Holmes, M.: The gelatinous nature of the sea-surface microlayer, Mar. Chem., 110, 89-97, 2008.

Zhou, J., Mopper, K., and Passow, U.: The role of surface-active carbohydrates in the formation of transparent exopolymer particles (TEP) by bubble adsorption of seawater, Limnol. Oceanogr., 43, 1860-1871, 1998. 